\section{La réponse inhabituelle des noyaux de cellules souches embryonnaires aux forces mécaniques}

Alexandre J. Kabla ${ }^{1}$, Kevin Chalut ${ }^{2}$

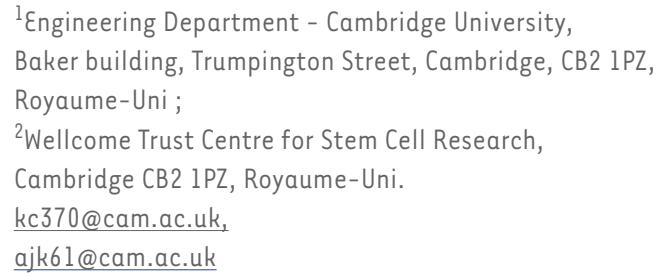

${ }^{1}$ Engineering Department - Cambridge University, Royaume-Uni ;

'Wellcome Trust Centre for Stem Cell Research, Cambridge CB2 1PZ, Royaume-Uni.

ajk61@cam.ac.uk

> La biophysique du cytosquelette a connu un énorme essor au cours des dix dernières années. L'application à la biologie de concepts et de méthodes expérimentales issus de la physique de la matière molle et de la physique statistique a permis d'élucider les paramètres physiques contrôlant la dynamique du cytosquelette. En fonction de l'échelle de temps, ce dernier peut se comporter comme un réseau de fibres élastiques (rapide) ou comme un matériau à forte plasticité (lent) [1]. L'analyse biochimique du système a, par ailleurs, permis l'identification des acteurs moléculaires contrôlant l'architecture de ce matériau. L'approche multidisciplinaire combinant physique, chimie et biologie a fondamentalement transformé notre vision du cytosquelette, de sa structure, de ses fonctions, et a eu un impact considérable sur notre compréhension de l'anatomie des cellules [2], de leurs mécanismes de migration [3], ainsi que de la morphogenèse animale [4].

\section{Quel rôle pour les liens}

\section{entre cytosquelette et noyau}

Les principaux rôles physiologiques du cytosquelette - maintien de l'intégrité cellulaire, migration, transport moléculaire - expliquent bien ses fortes interactions avec la membrane cellulaire. II semble en revanche surprenant dans ce contexte que le cytosquelette soit également intimement relié au noyau cellulaire, en particulier par l'intermédiaire des nesprines, lamines et des complexes
LINC (linker of nucleoskeleton and cytoskeleton) $[5,6]$. Pourquoi la machinerie génétique de la cellule [7] serait-elle couplée aux forces du monde extracellulaire? Cette question se pose encore plus dans le cas des cellules souches embryonnaires (CSE), qui n'expriment pas les lamines $A$ et $C[8]$, responsables de l'intégrité mécanique du noyau [9]. Si la cellule était simplement contrainte de suivre un programme génétique régulé par des signaux moléculaires, pourquoi la forme du noyau serait-elle influencée par le cytosquelette? On peut imaginer que la mécanotransduction, c'est-à-dire la capacité qu'a la cellule d'ajuster son comportement en fonction de son environnement mécanique, ait un rôle à jouer. Une hypothèse serait par exemple que le cytosquelette, en transmettant les forces de la membrane au noyau, influence l'organisation de la chromatine et, par là même, l'expression génétique. Avec l'essor récent des techniques de capture de configurations chromosomiques [10, $11,15]$, c'est à présent un moment clé pour la biophysique du noyau cellulaire; tout comme dans le cas du cytosquelette, l'objectif premier est de considérer ce système en tant que matériau et cesser de ne le regarder que comme dépositaire de l'information génétique.

\section{Comportement auxétique inhabituel du noyau de CSE soumises à des forces mécaniques \\ C'est pour cela que nous avons étudié la} réponse des noyaux de cellules souches embryonnaires aux forces mécaniques. En particulier, nous nous sommes intéressés au rôle des forces lors de la sortie de l'état pluripotent. Nous avons étudié des CSE de souris maintenues dans un état pluripotent naîf grâce à un inhibiteur particulier [12, 16]. Lorsqu'on les soustrait à cet inhibiteur, les CSE débutent leur différenciation. II faut approximativement un jour pour qu'elles quittent l'état de pluripotence et s'engagent dans une voie de différenciation. Il est alors possible de comparer les propriétés physiques de ces cellules, dites «de transition», avec celles des CSE naïves et celles des cellules différenciées ${ }^{1}[8]$.

Nous avons utilisé un microscope à force atomique pour comprimer des CSE naïves et des CSE de transition. Observé en coupe transversale, le noyau de ces dernières diminue de taille en réponse à une compression longitudinale. Ce comportement est fortement contre-intuitif : en écrasant un matériau ordinaire, on s'attend à ce qu'il soit aplati par la force, en d'autres termes à ce qu'il s'élargisse en coupe transversale. C'est pourtant le contraire qui est observé dans le noyau des CSE de transition (Figure 1).

Nous avons alors confirmé ces résultats en effectuant des essais mécaniques en tension imposés au noyau par l'intermédiaire du cytosquelette. Pour cela, nous

\footnotetext{
Davantage de détails concernant la définition de ces états sont disponibles dans notre étude publiée récemment dans
} Nature Materials [8]. 


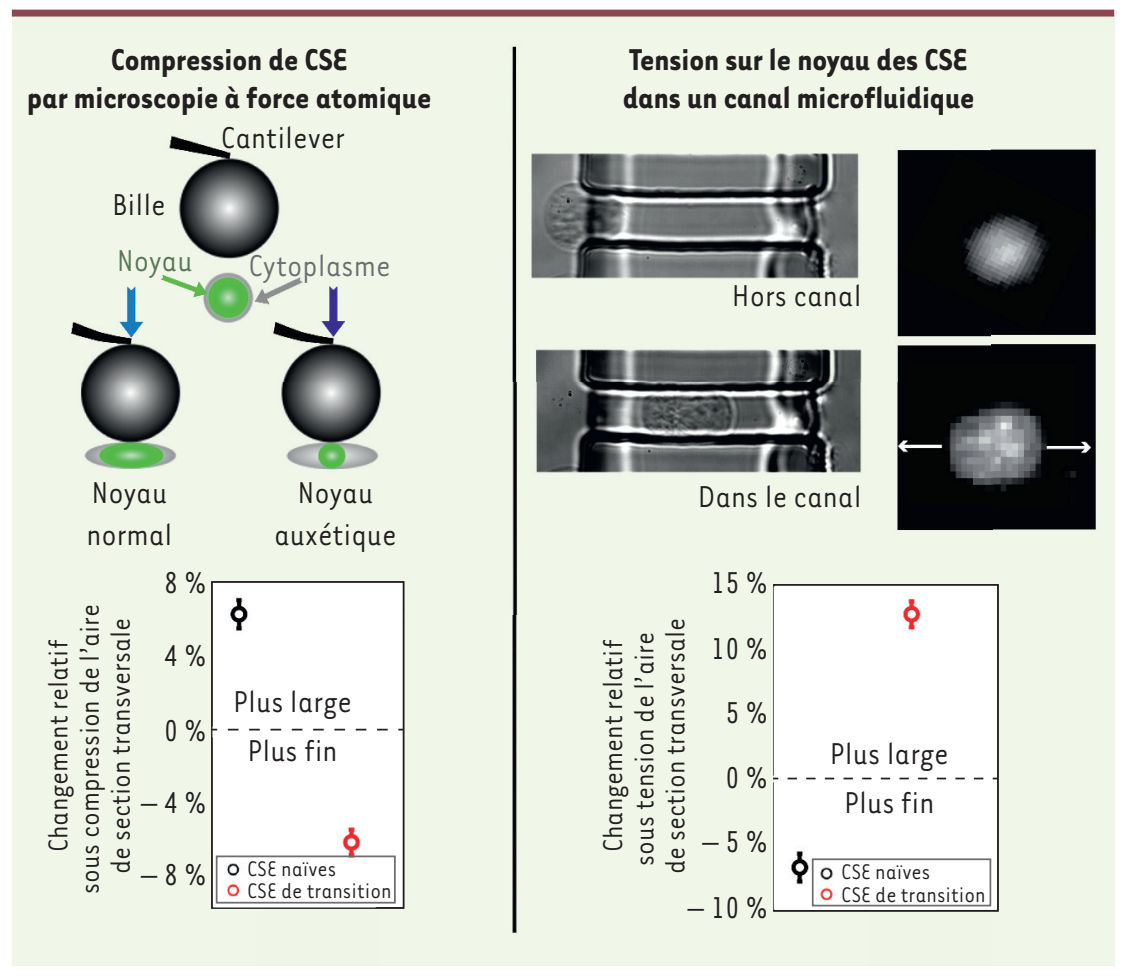

Figure 1. Deux mesures indépendantes des propriétés auxétiques. À gauche, les résultats des tests en compression par microscope à force atomique. Les données sur le changement relatif de l'aire de section transversale du noyau montrent que les noyaux des cellules pluripotentes naïves s'aplatissent sous compression, tandis que ceux des cellules pluripotentes « de transition » se contractent transversalement. À droite, les résultats obtenus dans les canaux microfluidiques montrent que les noyaux des cellules de transition se dilatent transversalement sous l'effet d'une tension induite par l'élongation du cytoplasme, contrastant avec la réponse des noyaux de cellules naïves.

avons développé un système microfluidique comportant des canaux dont la section transversale est plus petite que les CSE, mais plus grande que leur noyau, en moyenne. À l'entrée du canal, le cytoplasme doit alors s'allonger et impose en conséquence des forces de tension sur le noyau. Pour un matériau ordinaire, une tension longitudinale se traduit par une diminution de l'aire de section transversale; tirer sur un élastique réduit ainsi sa section. Dans le cas des CSE de transition, nous avons observé le contraire, conformément aux résultats obtenus à l'aide du microscope à force atomique. Lors du passage dans le canal, le volume des noyaux de CSE pouvait augmenter de plus de $50 \%$ (Figure 1).

Cette propriété inhabituelle du noyau doit trouver son origine dans la structure qui est contraire à l'idée communément admise selon laquelle c'est la chromatine des CSE naïves qui est dans un état de condensation minimale.

Propriété auxétique du noyau : un moyen de réguler la concentration protéique

Un matériau qui s'étend transversalement sous tension et se contracte sous compression est dit auxétique [14]. C'est une propriété très inhabituelle, en particulier parmi les matériaux biologiques. L'utilisation de matériaux auxétiques est cependant envisagée pour de nombreuses applications, du fait de leur capacité à absorber les chocs (gilets pare-balles, isolation sonore, etc.) ou à catalyser certaines réactions. II est donc légitime de s'interroger sur le rôle physiologique d'une telle propriété du noyau des CSE. Une conséquence directe de cette propriété auxétique est le couplage entre tension mécanique et absorption de fluide due à l'augmentation de volume. Afin d'identifier des mouvements de fluides, nous avons utilisé des cellules imprégnées de fluorescéine qui possèdent par défaut une plus grande concentration de fluorophore dans le cytoplasme que dans le noyau. En tirant sur le noyau de CSE, nous avons pu alors montrer que la fluorescéine se déplaçait dans le noyau des CSE de transition, mais pas dans celui des CSE naïves. Ce comportement des noyaux auxétiques permet ainsi à une contrainte mécanique de causer un changement très significatif de la concentration des protéines du noyau, offrant de ce fait un mécanisme simple de mécanotransduction. Un changement de densité du noyau en fonction de son état de tension ou compression implique également un changement de rigidité qui pourrait être impliqué dans d'autres processus morphogénétiques, comme la ségrégation des cellules au sein des tissus.

Nous sommes encore très loin d'une compréhension profonde des mécanismes et implications du comportement auxétique des noyaux de CSE. 
Ce travail est cependant un exemple d'approche multidisciplinaire appliquée à la biologie. La découverte d'un comportement auxétique dans le noyau de certaines cellules contribuera à long terme à mieux comprendre les rôles respectifs des signalisations, moléculaire et mécanique, sur la pluripotence, la différenciation et plus généralement sur le développement embryonnaire. $\diamond$

Auxetic nuclei in embryonic stem cells

\section{LIENS D'INTÉRÊT}

Les auteurs déclarent n'avoir aucun lien d'intérêt concernant les données publiées dans cet article.

\section{RÉFÉRENCES}

1. Deng L, Trepat X, Butler JP, et al. Fast and slow dynamics of the cytoskeleton. Nat Mater 2006 ; $5: 636-40$.
2. Paluch $\varepsilon$, Heisenberg CP. Biology and physics of cell shape development in the cytoskeleton. Curr Biol 2009 ; 19 : R790-9.

3. Gardel ML, Schneider IC, Aratyn-Schaus Y, Waterman CM. Mechanical integration of actin and adhesion dynamics in cell migration. Annu Rev Cell Dev Biol $2010 ; 26: 315-33$

4. Lecuit T, Lenne PF, Munro $\varepsilon$. Force generation, transmission, and integration during cell and tissue morphogenesis. Annu Rev Cell Dev Biol 2011 ; 27 : 157-84.

5. Lombardi ML, Jaalouk DE, Shanahan CM, et al. The interaction between nesprins and sun proteins at the nuclear envelope is critical for force transmission between the nucleus and cytoskeleton. J Biol Chem $2011 ; 286$ : 26743-53.

6. Swift J, Ivanovska IL, Buxboim A. et al. Nuclear lamin-A scales with tissue stiffness and enhances matrix-directed differentiation. Science 2013 ; $341: 1240104$.

7. Kind J, Pagie L, Ortabozkoyun H, et al. Single-cell dynamics of genome-nuclear lamina interactions. Cell 2013 ; 153 : 178-92.

8. Pagliara S, Franze K, McClain CR, et al. Auxetic nuclei in embryonic stem cells exiting pluripotency. Nat Mater $2014 ; 13: 638-44$.
9. Pajerowski JD, Dahl KN, Zhong FL, et al. Physical plasticity of the nucleus in stem cell differentiation. Proc Natl Acad Sci USA 2007 ; 104 : 15619-24.

10. Bickmore WA, van Steensel B. Genome architecture: domain organization of interphase chromosomes. Cell $2013 ; 152: 1270-84$

11. Nagano $T$, Lubling $Y$, Stevens TJ, et al. Single-cell $\mathrm{Hi}-\mathrm{C}$ reveals cell-to-cell variability in chromosome structure. Nature $2013 ; 502: 59-64$

12. Ying QL, Wray J, Nichols J, et al. The ground state of embryonic stem cell self-renewal. Nature 2008 ; 453, 519-23.

13. Chalut KJ, Höpfler M, Lautenschläger $F$, et al. Chromatin decondensation and nuclear softening accompany Nanog downregulation in embryonic stem cells. Biophys J $2012 ; 103,2060-70$.

14. K. E. Evans and A. Alderson. Auxetic materials: Functional materials and structures from lateral thinking! Adv Mater $2000 ; 12: 617-23$

16. Kieffer $\varepsilon$, Kuntz $S$, Viville $S$. Tour d'horizon des lignées de cellules souches pluripotentes. Med Sci (Paris) $2010 ; 26: 848-54$.

15. Umlauf $D$. Le génome intime... et en trois dimensions. Med Sci (Paris) 2014 (sous presse).

\section{NOUVELL\&}

\section{L'activité neuronale influence le développement des vaisseaux sanguins du cerveau}

Baptiste Lacoste
Dr Chenghua Gu Laboratory,

Department of Neurobiology, Harvard Medical School, 220 Longwood Avenue, MA02115, Boston, États-Unis.

baptiste_lacoste@hms.harvard.edu

vité neuronale et l'apport énergétique. Les interactions neurovasculaires sont bien connues de nos jours pour leur implication dans la régulation du débit sanguin cérébral [4, 5], que reflète l'imagerie cérébrale fonctionnelle par résonance magnétique (IRM fonctionnelle, ou IRM $M_{f}$ ). Le substrat anatomique des interactions neurovasculaires est appelé « unité neurovasculaire », un système multicellulaire complexe où neurones, astrocytes, péricytes et endothélium collaborent pour assurer le bon fonctionnement du cerveau (Figure 1A-B). Le couplage neurovasculaire est en effet à l'origine des signaux détectés en IRM $M_{f}$, signaux qui permettent d'observer l'activation d'une zone cérébrale lors d'une tâche particulière. C'est l'apport de sang dans cette zone activée (plus précisément un changement 